九州大学学術情報リポジトリ

Kyushu University Institutional Repository

Resonance and web structure in discrete soliton systems: the two-dimensional Toda lattice and its fully discrete and ultra-discrete analogues

Maruno, Ken-ichi

Faculty of Mathematics, Kyushu University

Biondini, Gino

Department of Mathematics, Ohio State University

http://hdl. handle. net/2324/11835

出版情報: Journal of physics. A, Mathematical and general. 37 (49), pp.11819-11839, 2004-1210. Institute of Physics

バージョン:

権利関係: 


\section{MHF Preprint Series}

Kyushu University

21st Century COE Program

Development of Dynamic Mathematics with

High Functionality

Resonance and web structure in

discrete soliton systems: the two-dimensional Toda lattice and its fully discrete and ultra-discrete versions

K. Maruno \& G. Biondini

MHF 2004-16

( Received June 2, 2004 )

Faculty of Mathematics

Kyushu University

Fukuoka, JAPAN 


\title{
Resonance and web structure in discrete soliton systems: the two-dimensional Toda lattice and its fully discrete and ultra-discrete versions
}

\author{
Ken-ichi Maruno ${ }^{1} \dagger$ and Gino Biondini ${ }^{2}$ \\ ${ }^{1}$ Faculty of Mathematics, Kyushu University, Hakozaki, Higashi-ku, Fukuoka, 812-8581, \\ Japan \\ 2 Department of Mathematics, Ohio State University, 231 West 18th Ave, Columbus, OH \\ 43210, USA
}

\begin{abstract}
We present a class of solutions of the two-dimensional Toda lattice equation, its fully discrete analogue and its ultra-discrete limit. These solutions demonstrate the existence of soliton resonance and web-like structure in discrete integrable systems such as differentialdifference equations, difference equations and cellular automata (ultra-discrete equations).
\end{abstract}

2 June 2004

PACS numbers: 02.30.Jr, 05.45.Yv

To be submitted to : J. Phys. A: Math. Gen.

\section{Introduction}

The discretization of integrable systems is an important issue in mathematical physics. A discretization process in which the dependent variables are discretized in addition to the independent variables is known as "ultra-discretization". One of the most important ultradiscrete soliton systems is the so-called "soliton cellular automaton" (SCA) [12, 16, 17]. A general method to obtain the SCA from discrete soliton equations was proposed in Refs. $[6,18]$ and involves using an appropriate limiting procedure.

Another issue which has received renewed interest in recent years is the phenomenon of soliton resonance, which was first discovered for the Kadomtsev-Petviashvili (KP) equation [8] (see also Refs. [7, 11]). More general resonant solutions possessing a web-like structure have recently been observed in a coupled KP (cKP) system [4, 5] and for the KP equation itself [1]. In particular, the Wronskian formalism was used in Ref. [1] to classify a class of resonant solutions of KP which also satisfy the Toda lattice hierarchy. It was also conjectured in Ref. [1] that resonance and web structure are not limited to KP and cKP, but rather they are a generic feature of integrable systems whose solutions can be expressed in terms of Wronskians.

The aim of this paper is to study soliton resonance and web structure in discrete soliton systems. In particular, by studying a class of soliton solutions of the two-dimensional Toda lattice (2DTL) equation, of its fully discrete version, and of their ultra-discrete analogue which

† E-mail: maruno@math.kyushu-u.ac.jp. 
was recently introduced by Nagai et al $[9,10]$, we show that an analogue to the class of solutions studied in Ref. [1] can be defined for all three of these systems, and that a similar type of resonant solutions with web-like structure is produced as a result in all three of these systems. To our knowledge, this is the first time that resonant behavior and web structure are observed in discrete soliton systems. These results also confirm that soliton resonance and web-like structure are general features of two-dimensional integrable systems whose solutions can be expressed via the determinant formalism.

\section{Resonance and web structure in the two-dimensional Toda lattice equation}

We start by considering the two-dimensional Toda lattice (2DTL) equation,

$$
\frac{\partial^{2}}{\partial x \partial t} Q_{n}=V_{n+1}-2 V_{n}+V_{n-1},
$$

with $Q_{n}(x, t)=\log \left[1+V_{n}(x, t)\right]$. Equation (2.1) can be written in bilinear form

$$
\frac{\partial^{2} \tau_{n}}{\partial x \partial t} \tau_{n}-\frac{\partial \tau_{n}}{\partial t} \frac{\partial \tau_{n}}{\partial x}=\tau_{n+1} \tau_{n-1}-\tau_{n}^{2}
$$

through the transformation

$$
V_{n}(x, t)=\frac{\partial^{2}}{\partial x \partial t} \log \tau_{n}(x, t) .
$$

It is well-known that some solutions of the 2DTL equation can be written via the Casorati determinant form $\tau_{n}=\tau_{n}^{(M)}$ [3], with

$$
\tau_{n}^{(M)}=\left|\begin{array}{ccc}
f_{n}^{(1)} & \cdots & f_{n+M-1}^{(1)} \\
\vdots & \ddots & \vdots \\
f_{n}^{(M)} & \cdots & f_{n+M-1}^{(M)}
\end{array}\right|
$$

where $\left\{f_{n}^{(1)}(x, t), \ldots, f_{n}^{(M)}(x, t)\right\}$ is a set of $M$ linearly independent solutions of the linear equations

$$
\frac{\partial f_{n}}{\partial x}=f_{n+1}, \quad \frac{\partial f_{n}}{\partial t}=-f_{n-1},
$$

for $1 \leq i \leq M$. For example, a two-soliton solution of the 2DTL is obtained by the set $\left\{f^{(1)}, f^{(2)}\right\}$, with

$$
f_{n}^{(i)}=\mathrm{e}^{\theta_{n}^{(2 i-1)}}+\mathrm{e}^{\theta_{n}^{(2 i)}}, \quad i=1,2,
$$

where the phases $\theta^{(j)}$ are given by linear functions of $(n, x, t)$,

$$
\theta_{n}^{(j)}(x, t)=n \log p_{j}+p_{j} x-\frac{1}{p_{j}} t+\theta_{0}^{(j)}, \quad j=1, \ldots, 4,
$$

with $p_{1}<p_{2}<p_{3}<p_{4}$. Equation (2.6) can be extended to the $M$-soliton solution with $\left\{f^{(1)}, \cdots, f^{(M)}\right\}$.

On the other hand, solutions of the 2DTL equation can also be obtained by the set of $\tau$ functions $\left\{\tau_{n}^{(M)} \mid M=1, \ldots, N\right\}$ with the choice of $f$-functions,

$$
f_{n}^{(i)}=f_{n+i-1}, \quad 1<i \leq M \leq N,
$$

with

$$
f_{n}=\sum_{j=1}^{N} \mathrm{e}^{\theta_{n}^{(j)}} .
$$



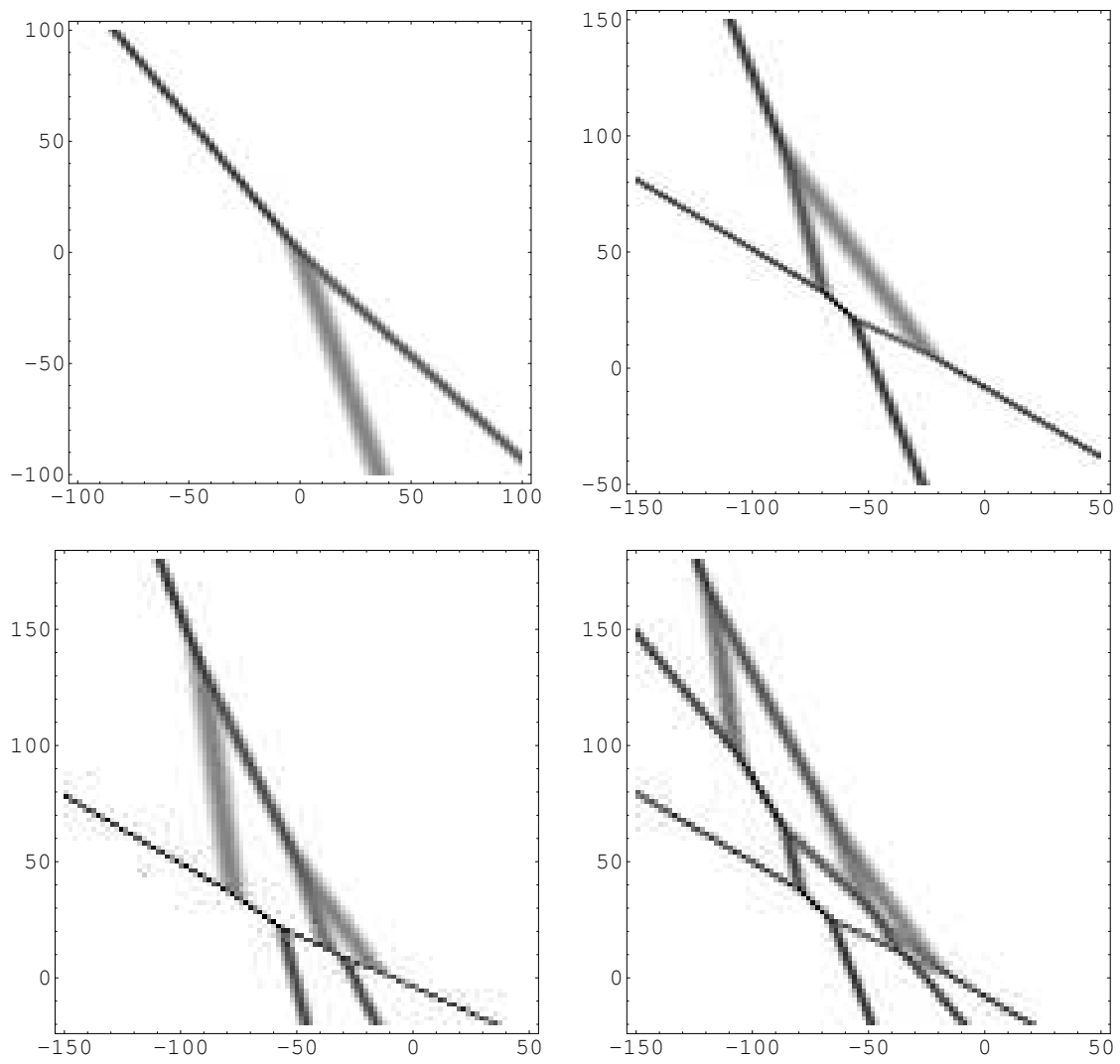

Figure 1. Resonant solutions of the two-dimensional Toda lattice: (a) $(2,1)$-soliton solution (i.e., a Y-junction) at $t=0$, with $M=1, N=3, p_{1}=1 / 4, p_{2}=1 / 2, p_{3}=2$; (b) (2,2)soliton solution at $t=14$, with $M=2, N=4, p_{1}=1 / 8, p_{2}=1 / 2, p_{3}=1$ and $p_{4}=4$; (c) (3,2)-soliton solution at $t=10$, with $M=2, N=5, p_{1}=1 / 10, p_{2}=1 / 5, p_{3}=1 / 2$, $p_{4}=1$ and $p_{5}=6$; (d) (3,3)-soliton solution at $t=14$, with $M=3, N=6, p_{1}=1 / 10$, $p_{2}=1 / 4, p_{3}=1 / 2$ and $p_{4}=1, p_{5}=2, p_{6}=4$. In all cases the horizontal axis is $n$ and the vertical axis is $x$, and each figure is a plot of $q(n, x, t)$ in logarithmic grayscale. Note that the values of $n$ in the horizontal axis are discrete.

and with the phases $\theta_{n}^{(j)}, 1 \leq i \leq N$ still given by Eq. (2.7). If the $f$-functions are chosen according to eq. (2.8), the $\tau$ function $\tau_{n}^{(M)}$ is then given by the Hankel determinant

$$
\tau_{n}^{(M)}=\left|\begin{array}{ccc}
f_{n} & \cdots & f_{n+M-1} \\
\vdots & \ddots & \vdots \\
f_{n+M-1} & \cdots & f_{n+2 M-2}
\end{array}\right|,
$$

for $1 \leq M \leq N$. It should be noted that, even when the set of functions $\left\{f_{n}^{(i)}\right\}_{i=1}^{M}$ is chosen according to Eq. (2.8), no derivatives appear in the $\tau$ function, and therefore Eq. (2.10) cannot be considered a Wronskian in the same sanse as for the KP equation (cf. Eq. (1.9) in Ref. [1]). Nonetheless, this choice produces a similar outcome as in the KP equation. Indeed, similarly to Ref. [1], we have the following: 
Lemma 2.1 Let $f_{n}$ be given by Eq. (2.9), with $\theta_{n}^{(j)}(j=1, \ldots, N)$ given by Eq. (2.7). Then, for $1 \leq M \leq N-1$ the $\tau$ function defined by the Hankel determinant (2.10) has the form

$$
\tau_{n}^{(M)}=\sum_{1 \leq i_{1}<\cdots<i_{M} \leq N} \Delta\left(i_{1}, \ldots, i_{M}\right) \exp \left(\sum_{j=1}^{M} \theta_{n}^{\left(i_{j}\right)}\right),
$$

where $\Delta\left(i_{1}, \ldots, i_{M}\right)$ is the square of the van der Monde determinant,

$$
\Delta\left(i_{1}, \ldots, i_{M}\right)=\prod_{1 \leq j<l \leq M}\left(p_{i_{j}}-p_{i_{l}}\right)^{2} .
$$

Proof. Apply the Binet-Cauchy theorem to Eq. (2.10), as in Ref. [1].

An immediate consequence of Lemma 2.1 is that the $\tau$ function $\tau_{n}^{(M)}$ is positive definite, and therefore all the solutions generated by it are non-singular. Like its analogue in the KP equation [1], the above $\tau$ function produces soliton solutions of resonant type with web structure. More precisely, we conjecture that, like its analogue in the KP equation, the above $\tau$ function produces an $\left(N_{-}, N_{+}\right)$-soliton solution, that is, a solution with $N_{-}=N-M$ asymptotic line solitons as $x \rightarrow-\infty$ and $N_{+}=M$ asymptotic line solitons as $x \rightarrow \infty$. As an example, in Fig. 1 we show a $(2,1)$-soliton solution (also called a Y-shape solution, or a Y-junction), a (2,2)-soliton solution, a (2,3)-soliton solution and a (3,3)-soliton solution.

Note that even when $N_{+}=N_{-}=M$, the interaction pattern of resonant soliton solutions differs from that of ordinary $M$-soliton solutions. As seen from Fig. 1, the resonant solutions of the 2DTL obtained from Eq. (2.10) are very similar to the solitons of the KP and coupled KP equation $[1,4,5]$, where such solutions were called "spider-web" solitons. (In contrast, an ordinary $M$-soliton solution produces a simple pattern of $M$ intersecting lines.) The web structure manifests itself in the number of bounded regions, the number of vertices and the number of intermediate solitons, which are respectively $\left(N_{-}-1\right)\left(N_{+}-1\right)$, $2 N_{-} N_{+}-N$ and $3 N_{-} N_{+}-2 N$ for an $\left(N_{-}, N_{+}\right)$-soliton solution [1]. (In contrast, an ordinary $M$-soliton solution has $(M-1)(M-2) / 2$ bounded regions and $M(M-1) / 2$ interaction vertices.) Finally, it should be noted that, as in the KP equation, only the Y-shape solution is a traveling wave solution. All other resonant solutions (as well as ordinary $M$ soliton solutions with $M \geq 3$ ) have a time-dependent shape, as shown in Ref. [1].

\section{Resonance and web structure in the fully discrete 2D Toda lattice equation}

We now consider a fully discrete analogue of the 2DTL equation (2.1), namely

$$
\begin{aligned}
& \Delta_{l}^{+} \Delta_{m}^{-} Q_{l, m, n}=V_{l, m-1, n+1}-V_{l+1, m-1, n}-V_{l, m, n}+V_{l+1, m, n-1}, \\
& V_{l, m, n}=(\delta \kappa)^{-1} \log \left[1+\delta \kappa\left(\exp Q_{l, m, n}-1\right)\right],
\end{aligned}
$$

with $l, m, n \in \mathbb{Z}, l$ and $m$ being the discrete analogues of the time $t$ and space $x$ coordinates, respectively, and where $\Delta_{l}^{+}$and $\Delta_{m}^{-}$are forward and backward difference operators defined by

$$
\begin{aligned}
\Delta_{l}^{+} f_{l, m, n} & =\frac{f_{l+1, m, n}-f_{l, m, n}}{\delta}, \\
\Delta_{m}^{-} f_{l, m, n} & =\frac{f_{l, m, n}-f_{l, m-1, n}}{\kappa} .
\end{aligned}
$$

Equation (3.1), which is the discrete analogue of Eq. (2.1), can be written in bilinear form [2] in a manner similar to Eq. (2.2):

$$
\begin{aligned}
\left(\Delta_{l}^{+} \Delta_{m}^{-} \tau_{l, m, n}\right) & \tau_{l, m, n}-\left(\Delta_{l}^{+} \tau_{l, m, n}\right) \Delta_{m}^{-} \tau_{l, m, n} \\
& =\tau_{l, m-1, n+1} \tau_{l+1, m, n-1}-\tau_{l+1, m-1, n} \tau_{l, m, n}
\end{aligned}
$$


with $Q_{l, m, n}$ related to $\tau_{l, m, n}$ by the transformation $V_{l, m, n}=\Delta_{l}^{+} \Delta_{m}^{-} \log \tau_{l, m, n}$, i.e.,

$$
Q_{l, m, n}=\log \frac{\tau_{l+1, m+1, n-1} \tau_{l, m, n+1}}{\tau_{l+1, m, n} \tau_{l, m+1, n}} .
$$

Note that $Q_{l, m, n}=\log \left[1+\left(\mathrm{e}^{\delta \kappa V_{l, m, n}}-1\right) / \delta \kappa\right]$. Special solutions of Eq. (3.4) (which is the discrete analogue of Eq. (2.2)) are obtained when the $\tau$ function $\tau_{l, m, n}$ is expressed in terms of a Casorati determinant $\tau_{l, m, n}=\tau_{l, m, n}^{(M)}$ as [2]

$$
\tau_{l, m, n}^{(M)}=\left|\begin{array}{cccc}
f_{l, m, n}^{(1)} & f_{l, m, n+1}^{(1)} & \cdots & f_{l, m, n+M-1}^{(1)} \\
f_{l, m, n}^{(2)} & f_{l, m, n+1}^{(2)} & \cdots & f_{l, m, n+M-1}^{(2)} \\
\vdots & \vdots & & \vdots \\
f_{l, m, n}^{(M)} & f_{l, m, n+1}^{(M)} & \cdots & f_{l, m, n+M-1}^{(M)}
\end{array}\right|
$$

where each of the functions $\left\{f_{l, m, n}^{(i)}, i=1,2, \cdots, M\right\}$ satisfies the following discrete dispersion relations:

$$
\begin{aligned}
& \Delta_{l}^{+} f_{l, m, n}=f_{l, m, n+1}, \\
& \Delta_{m}^{-} f_{l, m, n}=-f_{l, m, n-1} .
\end{aligned}
$$

If we take as a solution for Eqs. (3.7) and (3.8) the functions

with

$$
f_{l, m, n}^{(i)}=\phi\left(p_{i}\right)+\phi\left(q_{i}\right)
$$

$$
\phi(p)=p^{n}(1+\delta p)^{l}\left(1+\kappa p^{-1}\right)^{-m},
$$

the $\tau$ function (3.6) yields a $M$-soliton solution for the discrete 2DTL Eq. (3.4).

As in the continuous 2DTL, however, solutions of Eq. (3.4) can also be obtained when we consider the $\tau$ function defined by the Hankel determinant

$$
\tau_{l, m, n}^{(M)}=\left|\begin{array}{cccc}
f_{l, m, n} & f_{l, m, n+1} & \cdots & f_{l, m, n+M-1} \\
f_{l, m, n+1} & f_{l, m, n+2} & \cdots & f_{l, m, n+M} \\
\vdots & \vdots & & \vdots \\
f_{l, m, n+M-1} & f_{l, m, n+M} & \cdots & f_{l, m, n+2 M-2}
\end{array}\right|,
$$

where

$$
f_{l, m, n}=\sum_{i=1}^{N} \alpha_{i} \phi\left(p_{i}\right)
$$

which corresponds to choosing

$$
f_{l, m, n}^{(i)}=f_{l, m, n+i-1}
$$

for $i=1, \ldots, M$. Without loss of generality, we can label the parameters $p_{i}$ so that $0<p_{1}<p_{2}<\cdots<p_{N-1}<p_{N}$. Then, as in the continuous 2DTL, we have the following: Lemma 3.1 Let $f_{l, m, n}$ be given by Eq. (3.13). Then, for $1 \leq M \leq N-1$, the $\tau$ function defined by the Hankel determinant (3.11) has the form

$$
\tau_{n}^{(M)}=\sum_{1 \leq i_{1}<\cdots<i_{M} \leq N} \Delta\left(i_{1}, \ldots, i_{M}\right) \prod_{j=1}^{M} \alpha_{i_{j}} \phi\left(p_{i_{j}}\right)
$$

where $\Delta\left(i_{1}, \ldots, i_{M}\right)$ is the square of the van der Monde determinant,

$$
\Delta\left(i_{1}, \ldots, i_{M}\right)=\prod_{1 \leq j<l \leq M}\left(p_{i_{j}}-p_{i_{l}}\right)^{2},
$$



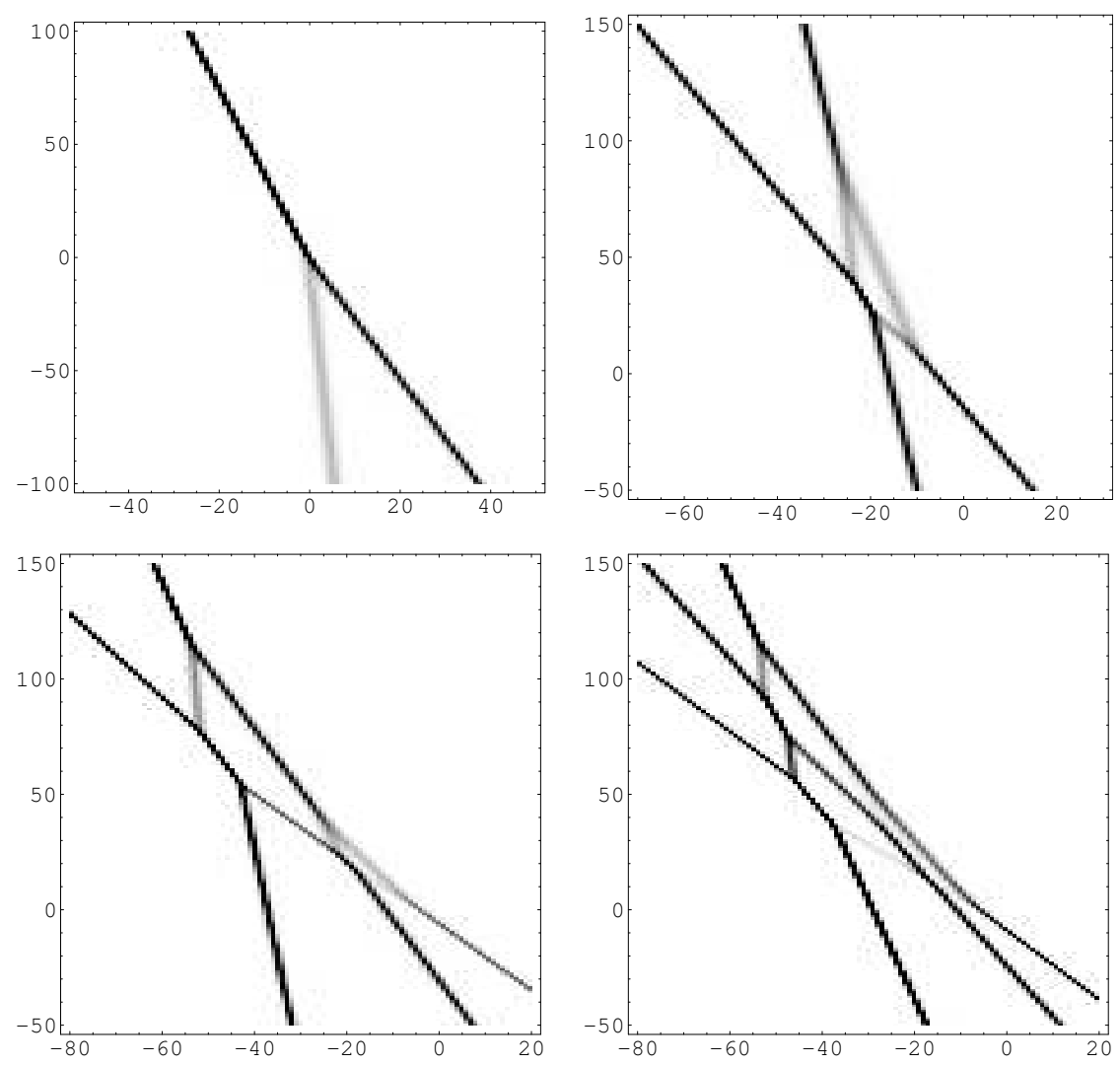

Figure 2. Resonant solutions of the fully discrete two-dimensional Toda lattice: (a) $(2,1)$ soliton solution (i.e., a Y-junction) at $l=0$, with $p_{1}=1 / 10, p_{2}=1 / 2, p_{3}=10$, (b) $(2,2)$ soliton solution at $t=40$, with $p_{1}=1 / 10, p_{2}=1 / 2, p_{3}=2, p_{4}=15$; (c) $(3,2)$-soliton solution at $t=80$, with $p_{1}=1 / 20, p_{2}=1 / 2, p_{3}=2, p_{4}=10, p_{5}=60$; (d) (3,3)-soliton solution at $t=80$, with $p_{1}=1 / 20, p_{2}=1 / 2, p_{3}=2, p_{4}=10, p_{5}=20, p_{6}=120$. In all cases $\delta=\kappa=1 / 4$; the horizontal axis is $n$ and the vertical axis is $n$, and each figure is a plot of $Q_{l, m, n}$ in logarithmic grayscale. Note that the values of both $m$ and $n$ in the horizontal and vertical axes are discrete.

Proof. Again, the result follows by applying the Binet-Cauchy theorem to the Hankel determinant (3.11).

Note that, unlike its counterpart in the continuous 2DTL, the $\tau$ function in Eq. (3.11) cannot be written in terms of a Wronskian, since no derivatives appear. However, as in the continuous 2DTL, the $\tau$ function thus defined is positive definite, and therefore all the solutions generated by it are non-singular. Like its analogue in the continuous 2DTL, the above $\tau$ function produces soliton solutions of resonant type with web structure, and we conjecture that, like in the continous case, an $\left(N_{-}, N_{+}\right)$-soliton with $N_{-}=N-M$ and $N_{+}=M$ is created. As an example, in Fig. 2 we show a $(2,1)$-soliton solution, a $(2,2)$ soliton solution, a $(2,3)$-soliton solution and a $(3,3)$-soliton solution. As in the continuous case, however, a full characterization of the solution remains a problem for further study. The resonant solutions of the fully discrete 2DTL provide the basis for the construction of the resonant solution of the ultra-discrete 2DTL, as is shown in the next two sections. 


\section{The ultra-discrete two-dimensional Toda lattice}

We now turn our attention to an ultra-discrete analogue of the 2DTL equation. Using Eqs. (3.2) and (3.3), we first write the 2DTL Eq. (3.4) in bilinear form as

$(1-\delta \kappa) \tau_{l+1, m, n} \tau_{l, m+1, n}-\tau_{l+1, m+1, n} \tau_{l, m, n}+\delta \kappa \tau_{l, m, n+1} \tau_{l+1, m+1, n-1}=0$,

We define the difference operator $\Delta^{\prime}$ as

$$
\Delta^{\prime}=\mathrm{e}^{-\partial_{n}}\left(\Delta_{n}^{+}-\Delta_{t}^{+}\right)\left(\Delta_{n}^{+}-\Delta_{m}^{+}\right),
$$

where from here on the symbols $\Delta_{l}^{+}, \Delta_{m}^{+}$and $\Delta_{n}^{+}$will be used to denote the difference operators

$$
\Delta_{l}^{+}=\mathrm{e}^{\partial_{l}}-1, \quad \Delta_{m}^{+}=\mathrm{e}^{\partial_{m}}-1, \quad \Delta_{n}^{+}=\mathrm{e}^{\partial_{n}}-1,
$$

and where the shift operators $\mathrm{e}^{\partial_{l}}, \mathrm{e}^{\partial_{m}}$ and $\mathrm{e}^{\partial_{n}}$ are defined by $\mathrm{e}^{\partial_{n}} f_{l, m, n}=f_{l, m, n+1}$ etc. That is,

$$
\Delta^{\prime} f_{l, m, n}=f_{l+1, m+1, n-1}+f_{l, m, n+1}-f_{l+1, m, n}-f_{l, m+1, n} .
$$

Using Eqs. (4.3) and (4.2), we can rewrite Eq. (4.1) as

$$
(1-\delta \kappa)+\delta \kappa \exp \left[\Delta^{\prime} \log \tau_{l, m, n}\right]=\exp \left[\Delta_{t}^{+} \Delta_{m}^{+} \log \tau_{l, m, n}\right],
$$

which becomes, taking a logarithm and applying $\Delta^{\prime}$ (assuming $\delta \kappa \neq 1$ ),

$$
\Delta^{\prime} \log \left[1+\frac{\delta \kappa}{1-\delta \kappa} \exp \left(\Delta^{\prime} \log \tau_{l, m, n}\right)\right]=\Delta_{t}^{+} \Delta_{m}^{+} \Delta^{\prime} \log \tau_{l, m, n} .
$$

We now take an ultra-discrete limit of Eq. (4.6) following Refs. (2.1) [9, 10]. This is accomplished by choosing the lattice intervals as

$$
\delta_{\varepsilon}=\mathrm{e}^{-r / \varepsilon}, \quad \kappa_{\varepsilon}=\mathrm{e}^{-s / \varepsilon},
$$

where $r, s \in \mathbb{Z}_{\geq 0}$ are some predetermined integer constants, and by defining

$$
v_{l, m, n}^{\varepsilon}=\Delta^{\prime} \varepsilon \log \tau_{l, m, n}^{\varepsilon},
$$

Taking the limit $\varepsilon \rightarrow 0^{+}$in Eq. (4.6) and noting that $\lim _{\varepsilon \rightarrow 0^{+}} \varepsilon \log \left(1+\mathrm{e}^{X / \varepsilon}\right)=\max (0, X)$, we then obtain

$$
\Delta_{t}^{+} \Delta_{m}^{+} v_{l, m, n}=\Delta^{\prime} \max \left(0, v_{l, m, n}-r-s\right),
$$

where $v_{l, m, n}=\lim _{\varepsilon \rightarrow 0^{+}} v_{l, m, n}^{\varepsilon}$. That is, using Eq. (4.8),

$$
v_{l, m, n}=\Delta^{\prime} \lim _{\varepsilon \rightarrow 0^{+}} \varepsilon \tau_{l, m, n}^{\varepsilon} .
$$

Equation (4.9) is the ultra-discrete analogue of the 2DTL equation, and can be considered a cellular automaton in the sense that $v_{l, m, n}$ takes on integer values.

Let us briefly discuss ordinary soliton solutions of the ultra-discrete 2DTL Eq. (4.9). As shown in Refs. [9, 10], soliton solutions for the ultra-discrete 2DTL Eq. (4.9) are obtained by an ultra-discretization of the soliton solution of the discrete 2DTL Eq. (3.4). For example, a one-soliton solution for Eq. (3.4) is given by

$$
\tau_{l, m, n}=1+\eta_{1}
$$

with

$$
\eta_{i}=\alpha_{i} \frac{\phi\left(p_{i}\right)}{\phi\left(q_{i}\right)}
$$

and where

$$
\phi(p)=p^{n}(1+\delta p)^{l}\left(1+\kappa p^{-1}\right)^{-m}
$$


as before. We introduce a new dependent variable

$$
\rho_{l, m, n}^{\varepsilon}=\varepsilon \log \tau_{l, m, n}
$$

and new parameters $P_{1}, Q_{1}, A_{1} \in \mathbb{Z}$ as

$$
\mathrm{e}^{P_{1} / \varepsilon}=p_{1}, \quad \mathrm{e}^{Q_{1} / \varepsilon}=q_{1}, \quad \mathrm{e}^{A_{1} / \varepsilon}=\alpha_{1} .
$$

Taking the limit $\varepsilon \rightarrow 0^{+}$, we then obtain

$$
\rho_{l, m, n}=\max \left(0, \Theta_{1}\right),
$$

where

$$
\begin{aligned}
\Theta_{i}= & A_{i}+n\left(P_{i}-Q_{i}\right)+l\left\{\max \left(0, P_{i}-r\right)-\max \left(0, Q_{i}-r\right)\right\} \\
& +m\left\{\max \left(0,-Q_{i}-s\right)-\max \left(0,-P_{i}-s\right)\right\},
\end{aligned}
$$

with $\mathrm{e}^{-r / \varepsilon}=\delta$ and $\mathrm{e}^{-s / \varepsilon}=\kappa$ as before, and where $\rho_{l, m, n}=\lim _{\varepsilon \rightarrow 0^{+}} \rho_{l, m, n}^{\varepsilon}$. According to Eqs. (4.10) and (4.14), the one-soliton solution for Eq. (4.9) is then given by

$$
v_{l, m, n}=\rho_{l+1, m+1, n-1}+\rho_{l, m, n+1}-\rho_{l+1, m, n}-\rho_{l, m+1, n} .
$$

Using a similar procedure we can construct a two-soliton solution. Equation (3.4) admits a two-soliton solution given by

$$
\tau_{l, m, n}=1+\eta_{1}+\eta_{2}+\theta_{12} \eta_{1} \eta_{2},
$$

with

$$
\theta_{12}=\frac{\left(p_{2}-p_{1}\right)\left(q_{1}-q_{2}\right)}{\left(q_{1}-p_{2}\right)\left(q_{2}-p_{1}\right)}
$$

and where $\eta_{i}=\alpha_{i} \phi\left(p_{i}\right) / \phi\left(q_{i}\right)$ as before $(i=1,2)$. In order to take the ultra-discrete limit of the above solution, we suppose without loss of generality that the soliton parameters satisfy the inequality

$$
0<p_{1}<p_{2}<q_{2}<q_{1} .
$$

Introducing again the dependent variable $\rho_{l, m, n}^{\varepsilon}=\varepsilon \log \tau_{l, m, n}$, as well as integer parameters $P_{i}, Q_{i}$ and $A_{i}$ as

$$
\mathrm{e}^{P_{i} / \varepsilon}=p_{i}, \quad \mathrm{e}^{Q_{i} / \varepsilon}=q_{i}, \quad \mathrm{e}^{A_{i} / \varepsilon}=\alpha_{i}
$$

$(i=1,2)$, and taking the limit of small $\varepsilon$, we obtain

$$
\rho_{l, m, n}=\max \left(0, \Theta_{1}, \Theta_{2}, \Theta_{1}+\Theta_{2}+P_{2}-Q_{2}\right),
$$

where $\Theta_{i}(i=1,2)$ was defined in Eq. (4.17), with $\rho_{l, m, n}=\lim _{\varepsilon \rightarrow 0^{+}} \rho_{l, m, n}^{\varepsilon}$ again, and where $v_{l, m, n}$ is obtained from $\rho_{l, m, n}$ using Eq. (4.18). Note that $P_{1}<P_{2}<Q_{2}<Q_{1}$.

More in general, starting from Eqs. (3.6) and (3.9) (with $0<p_{1}<p_{2}<\cdots<p_{N}<$ $\left.q_{N}<q_{N-1}<\cdots<q_{1}\right)$ and repeating the same construction, one obtains the $N$-soliton solution of the ultra-discrete 2DTL Eq. (4.9) as [10]

$$
\rho_{l, m, n}=\max _{\mu=0,1}\left[\sum_{1 \leq i \leq N} \mu_{i} \Theta_{i}+\sum_{1 \leq i<i^{\prime} \leq N} \mu_{i} \mu_{i^{\prime}}\left(P_{i^{\prime}}-Q_{i^{\prime}}\right)\right]
$$

where $\max _{\mu=0,1}$ indicates maximization over all possible combinations of the integers $\mu_{i}=$ 0,1 , with $i=1, \ldots, N$. Again, $v_{l, m, n}$ is obtained from $\rho_{l, m, n}$ via Eq. (4.18).

Ordinary soliton solutions corresponding to the above choices were presented in Ref. $[9,10]$. In the next section we show how this basic construction can be generalized to obtain resonant soliton solutions. 

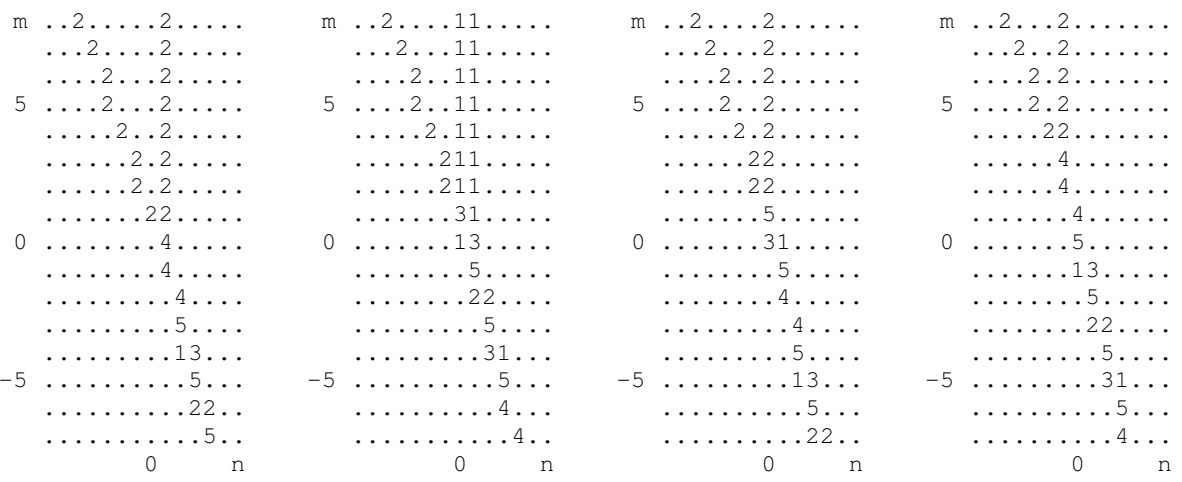

Figure 3. A $(2,1)$-resonant soliton solution (i.e., a Y-junction) for the ultradiscrete 2DTL Eq. (4.9), with $P_{1}=-5, P_{2}=1, P_{3}=4, r=3, s=1, A_{1}-A_{3}=1$, $A_{2}-A_{3}=1$ : (a) $l=0$, (b) $l=2$, (c) $l=4$, (d) $l=6$. The dots indicate zero values of $v_{l, m, n}$.

\section{Resonance and web structure in the ultra-discrete $2 \mathrm{D}$ Toda lattice equation}

Following Ref. [1], we now construct more general solutions of the ultra-discrete 2DTL equation (4.9) which display soliton resonance and web structure.

We first consider the case of a $(2,1)$-soliton for Eq. (3.4), which is given by

$$
\tau_{l, m, n}=\xi_{1}+\xi_{2}+\xi_{3}
$$

where

$$
\xi_{i}=\alpha_{i} \phi\left(p_{i}\right)
$$

$(i=1,2,3)$, with

$$
\phi(p)=p^{n}(1+\delta p)^{l}\left(1+\kappa p^{-1}\right)^{-m}
$$

as before, and where again we take $0<p_{1}<p_{2}<p_{3}$. As in the previous section, we introduce the new dependent variable

$$
\rho_{l, m, n}^{\varepsilon}=\varepsilon \log \tau_{l, m, n},
$$

and new parameters as

$$
\mathrm{e}^{P_{i} / \varepsilon}=p_{i}, \quad \mathrm{e}^{A_{i} / \varepsilon}=\alpha_{i}
$$

$(i=1,2,3)$, with $\mathrm{e}^{-r / \varepsilon}=\delta$ and $\mathrm{e}^{-s / \varepsilon}=\kappa$ as before. Taking the limit $\varepsilon \rightarrow 0^{+}$, we then obtain

$$
\rho_{l, m, n}=\max \left(R_{1}, R_{2}, R_{3}\right)
$$

where $\rho_{l, m, n}=\lim _{\varepsilon \rightarrow 0^{+}} \rho_{l, m, n}^{\varepsilon}$ as before, but where now

$$
R_{i}=A_{i}+n P_{i}+l \max \left(0, P_{i}-r\right)-m \max \left(0,-P_{i}-s\right)
$$

$(i=1,2,3)$. Figure 3 shows that this solution, which again can be called a $(2,1)$-soliton, is a Y-shape solution. Note however that the (2,1)-soliton in Fig. 3 looks like a $(1,2)$-soliton, in the sense that there are 2 solitons for large positive $m$ and only one for large negative $m$. In general, a $\left(N_{-}, N_{+}\right)$-soliton of the discrete 2DTL equation (3.1) leads to a $\left(N_{+}, N_{-}\right)$-soliton of Eq. (4.9) when taking the ultra-discrete limit. We also note that, interestingly, an L-shape solution can be is obtained instead of Y-shape solution for different solution parameters. An 

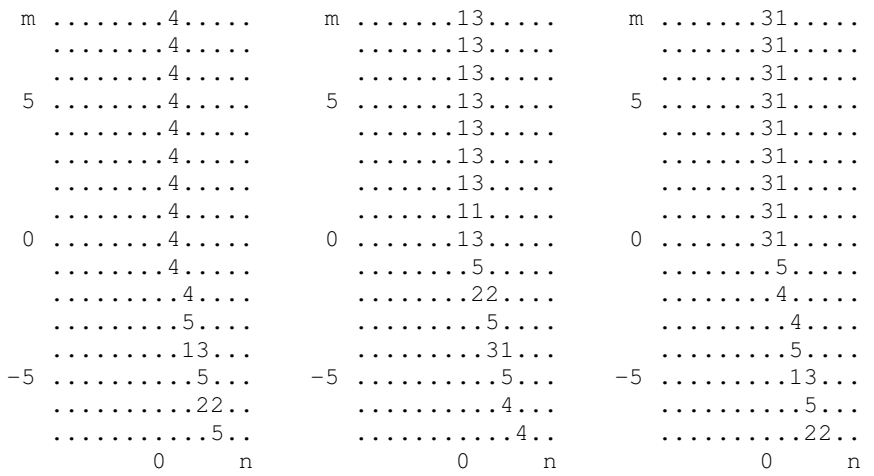
$m \ldots \ldots 4 \ldots \ldots$
$\ldots \ldots+\ldots \ldots$
$\ldots \ldots 4 \ldots . .$.
$5 \ldots \ldots .4 \ldots$
$\ldots \ldots 4 \ldots \ldots$
$\ldots \ldots 4 \ldots \ldots$
$\ldots \ldots 4 \ldots \ldots$
$\ldots . . . .4$
$\ldots \ldots+\ldots$
$\ldots \ldots 13 \ldots$
$\ldots \ldots \ldots$
$\ldots \ldots 22 \ldots$
$\ldots \ldots \ldots 5$
$-5 \ldots \ldots \ldots 31 \ldots$
$\ldots \ldots \ldots 5 \ldots$
$\ldots \ldots+\ldots$

Figure 4. An L-shape (2, 1)-resonant soliton solution for Eq. (4.9), with $P_{1}=-5, P_{2}=-1$, $P_{3}=4, r=3, s=1, A_{1}-A_{3}=1, A_{2}-A_{3}=1$ : (a) $l=0$, (b) $l=2$, (c) $l=4$, (d) $l=6$.

example of such a L-shape soliton is shown in Fig. 4. No analogue of this solution exists in the 2DTL and its fully discrete version.

Next, we consider the case of a $(2,2)$-soliton for Eq. (3.4) following Ref. [1]. Let us consider the following $\tau$ function

$$
\tau_{l, m, n}=\left|\begin{array}{cc}
f_{l, m, n} & f_{l, m, n+1} \\
f_{l, m, n+1} & f_{l, m, n+2}
\end{array}\right|
$$

where

$$
f_{l, m, n}=\xi_{1}+\xi_{2}+\xi_{3}+\xi_{4},
$$

where $\xi_{i}(i=1, \ldots, 4)$ is again defined as in Eq. (5.3), and where $0<p_{1}<p_{2}<p_{3}<p_{4}$ holds. We introduce again the new parameters $\mathrm{e}^{P_{k} / \varepsilon}=p_{k}$ and $\mathrm{e}^{A_{k} / \varepsilon}=\alpha_{k}(k=1, \ldots, 4)$ and the new dependent variable $\rho_{l, m, n}^{\varepsilon}=\varepsilon \log \tau_{l, m, n}$. Taking the limit $\varepsilon \rightarrow 0^{+}$, we then obtain

$$
\rho_{l, m, n}=\max _{1 \leq i<j \leq 4}\left(K_{i j}+2 P_{j}\right),
$$

where $\rho_{l, m, n}=\lim _{\varepsilon \rightarrow 0^{+}} \rho_{l, m, n}^{\varepsilon}$, as before, and

$$
K_{i j}=R_{i}+R_{j}
$$

and with $R_{j}$ given by Eq. (5.7) as before. Figure 5 shows the temporal evolution of a $(2,2)$ soliton solution. Note the appearance of a hole in Fig. 5.

Like in the two-dimensional Toda lattice (2.1) and its fully discrete version (3.1), we now consider more general resonant solutions for the ultra-discrete 2DTL (4.9). We start from the general $\tau$ function defined in Eq. (3.11), and introduce again the parameters $\mathrm{e}^{P_{k} / \varepsilon}=p_{k}$ and $e^{A_{k} / \varepsilon}=\alpha_{k}(k=1,2, \ldots, N)$ and the variable $\rho_{l, m, n}^{\varepsilon}=\varepsilon \log \tau_{l, m, n}$, together with $\mathrm{e}^{-r / \varepsilon}=\delta$ and $\mathrm{e}^{-s / \varepsilon}=\kappa$. Taking the limit $\varepsilon \rightarrow 0^{+}$, we then obtain the following solution of the ultra-discrete 2DTL (4.9):

$$
\rho_{l, m, n}=\max _{1 \leq i_{1}<\cdots<i_{M} \leq N}\left[\sum_{j=1}^{M} R_{i_{j}}+2 \sum_{j=2}^{M}(j-1) P_{i_{j}}\right],
$$

where again $\lim _{\varepsilon \rightarrow 0^{+}} \rho_{l, m, n}^{\varepsilon}=\rho_{l, m, n}$, with the maximum being taken among all possible combinations of the indices $i_{j}(j=1, \ldots, M)$, and where once more we have

$$
R_{i}=A_{i}+n P_{i}+l \max \left(0, P_{i}-r\right)-m \max \left(0,-P_{i}-s\right) .
$$



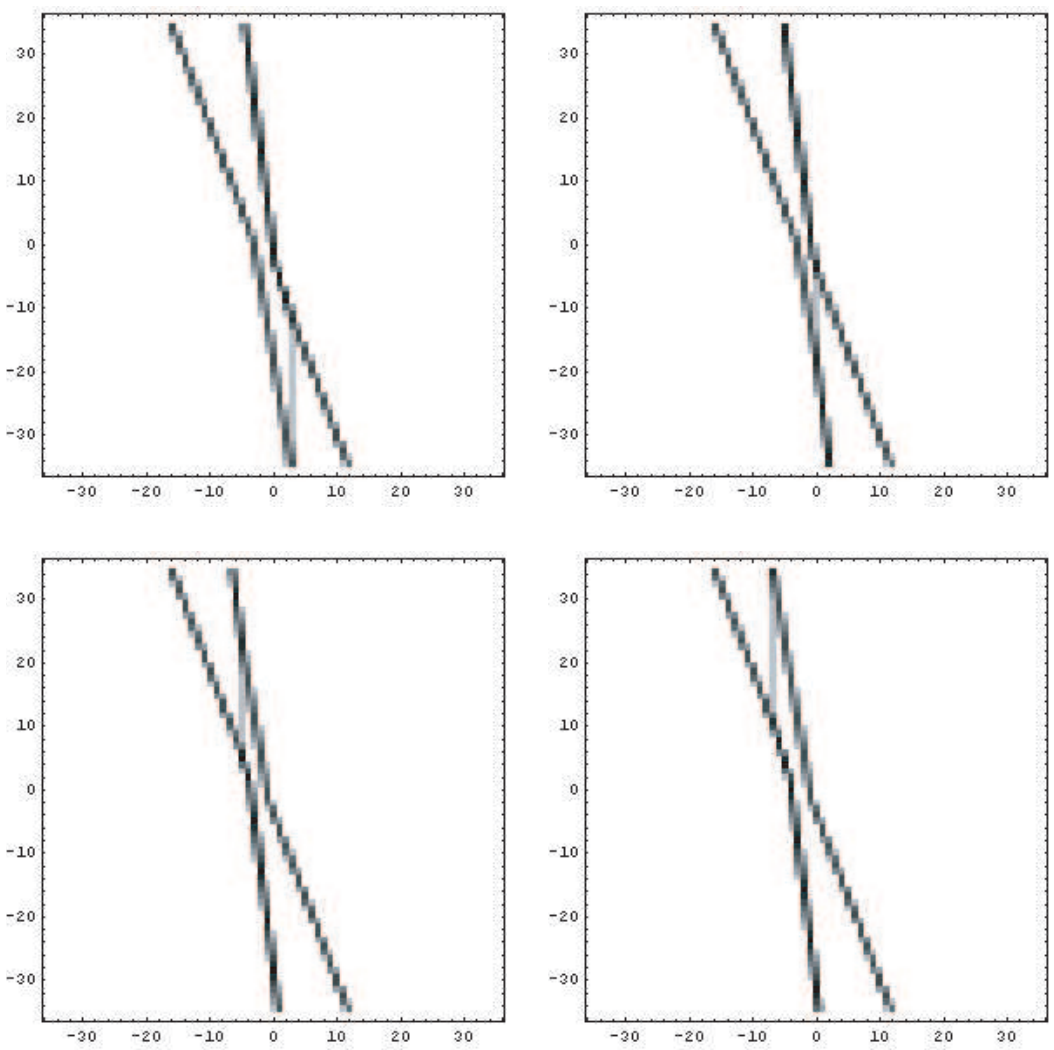

Figure 5. Snapshots illustrating the temporal evolution of a $(2,2)$-resonant soliton solution for Eq. (4.9), with $P_{1}=-7, P_{2}=-5, P_{3}=1, P_{4}=3, r=4, s=2, A_{1}=-10$, $A_{2}=-6, A_{3}=0, A_{4}=2$ : (a) $l=-12$; (b) $l=-7$; (c) $l=4$; (d) $l=7$. As in Figs. 3 and 4 , the horizontal axis is $n$ and the vertical axis is $m$. Since the interaction extends over a wider range of values of $m$ and $n$, the solution is now plotted in grayscale, in a similar way as in Figs. 1 and 2; the values of $v_{l, m, n}$ however are still discrete, as in Figs. 3 and 4.

Equation (5.12) produces complicated soliton resonance solutions. As an example, in Fig. 6 and Fig. 7 we show the snapshots of the time evolution of a $(3,3)$-resonant soliton solution and a $(4,4)$-resonant soliton solution. Indeed, as in the 2DTL and in its fully discrete analogue, we conjecture that Eq. (5.12) yields the $\left(N_{-}, N_{+}\right)$-soliton solution of the ultra-discrete 2DTL Eq. (4.9), with $N_{+}=M$ and $N_{-}=N-M$. It should be noted however that the interaction patterns in the ultra-discrete system differ somewhat from their analogues in the differential-difference and fully discrete cases. In particular, low-amplitude interaction arms may disappear when considering the ultra-discrete limit. Note also that the specific interactions which are produced in the ultra-discrete limit depend on the value of the parameters $r$ and $s$, and different kinds of solutions may appear for different values of $r$ and $s$. In particular, large values of $r$ and $s$ tend to result in the production of several vertical solitons, as shown in Figs. 6 and 7. In order to preserve the soliton count in these cases, all the outgoing vertical solitons should be counted as one, as should the incoming ones. In this sense, a set of outgoing or incoming vertical lines can be considered as a bound state composed of several solitons. A full characterization of these phenomena and their parameter dependence is however outside the scope of this work, and is a subject for future research. 

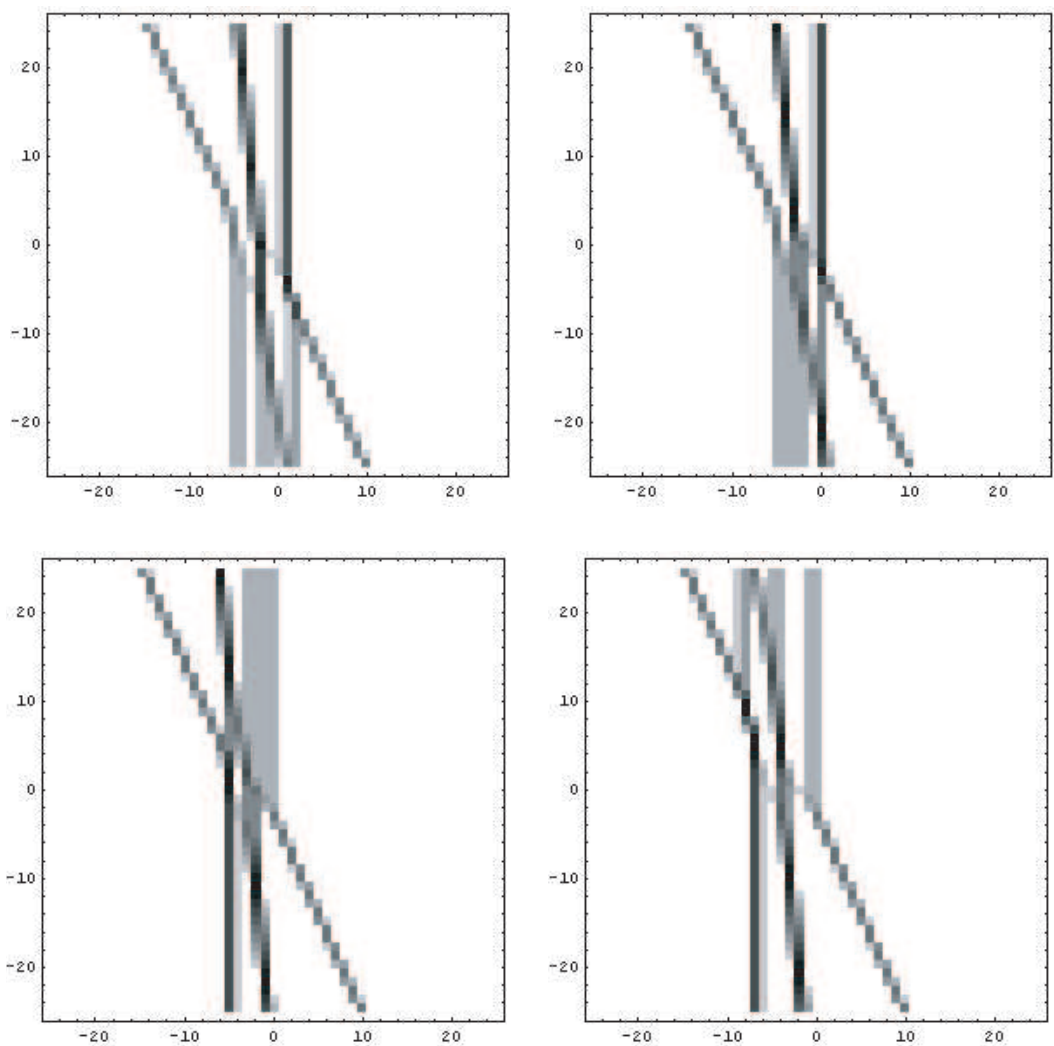

Figure 6. Snapshots illustrating the temporal evolution of a $(3,3)$-resonant soliton solution for Eq. (4.9), with $P_{1}=-10, P_{2}=-7, P_{3}=-5, P_{4}=-1, P_{5}=4, P_{6}=5, r=7$, $s=4, A_{1}=-8, A_{2}=-6, A_{3}=0, A_{4}=2, A_{5}=4, A_{6}=7:$ (a) $l=-15$, (b) $l=-10$, (c) $l=0$, (c) $l=10$.

\section{Conclusions}

We have demonstrated the existence of soliton resonance and web structure in discrete soliton systems by presenting a class of solutions of the two-dimensional Toda lattice (2DTL) equation, its fully discrete version and their ultra-discrete analogue. Soliton resonance and web structure had been previously found for nonlinear partial differential equations such as the $\mathrm{KP}$ and cKP systems. Since the 2DTL is a differential-difference equation, its fully discrete version a difference equation and their ultra-discrete version a cellular automaton, however our findings show that these phenomena are general features of two-dimensional integrable systems whose solutions are expressed in determinant form.

A full characterization of these solutions, including the study of asymptotic amplitudes and velocities, the resonance condition and the analysis of the intermediate patterns of interactions in all three cases is outside the scope of this work, and remains as a problem for further research. Of particular interest is the ultra-discrete 2DTL, where new types of solutions such as the L-shape soliton shown in Fig. 4 appear. We should also note that the solutions of the ultra-discrete 2DTL arise as a result of the properties of the maximum function, and therefore their study might require the use of techniques from tropical algebraic geometry, which is a subject of current research $[13,14,15]$. 

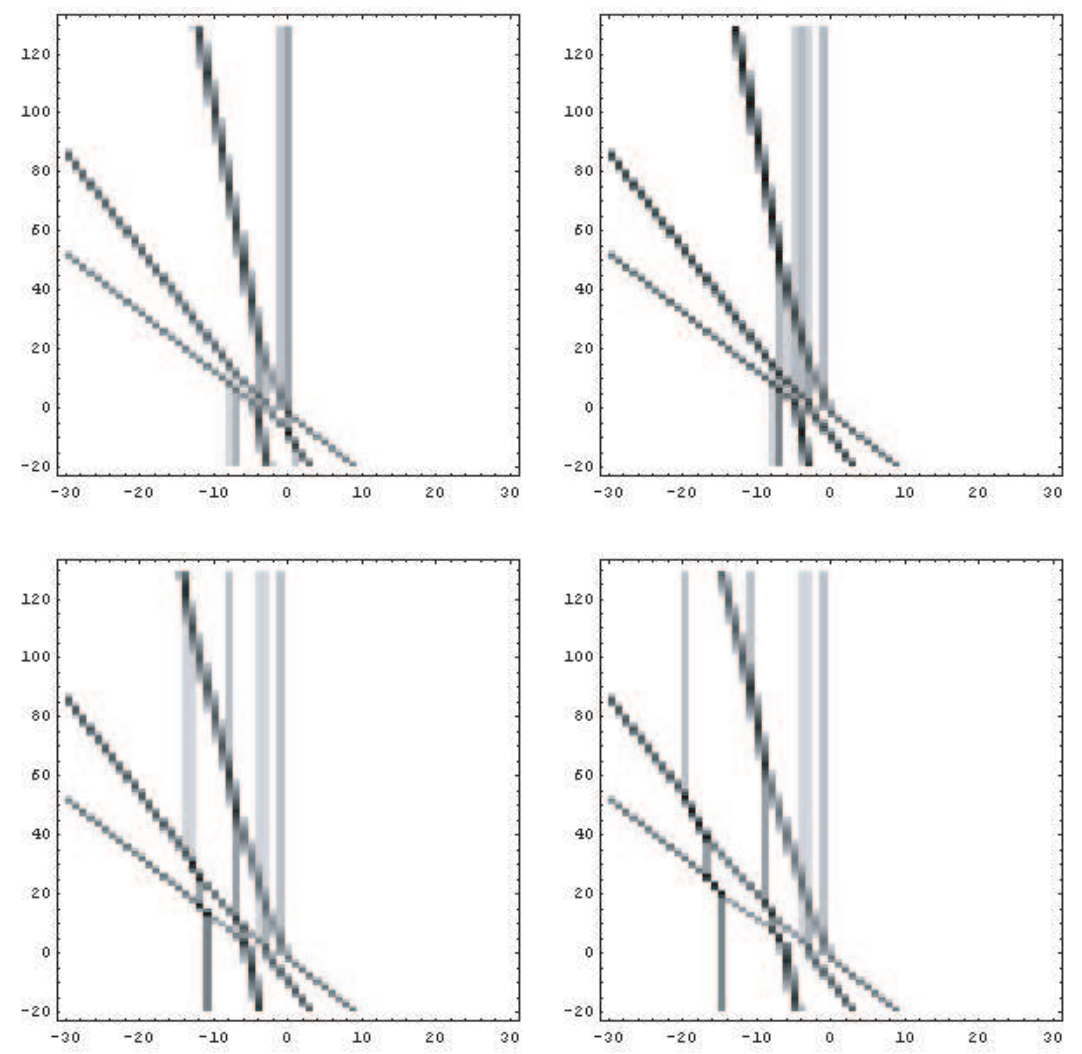

Figure 7. Snapshots illustrating the temporal evolution of a $(4,4)$-resonant soliton solution for Eq. (4.9), with $P_{1}=-15, P_{2}=-12, P_{3}=-9, P_{4}=-3, P_{5}=1, P_{6}=1, P_{7}=4$, $P_{8}=7, r=7, s=4, A_{1}=-8, A_{2}=-6, A_{3}=0, A_{4}=2, A_{5}=4, A_{6}=7, A_{7}=8$, $A_{8}=10$ : (a) $l=-10$, (b) $l=0$, (c) $l=10$, (d) $l=20$.

Finally, we note that the class of solutions presented in this work is just one of the possible choices that yield resonance and web structure. Just like with the KP and cKP equations, the class of soliton solutions of each of the systems we have considered (namely, the 2DTL and its fully discrete and its ultra-discrete analogues) is much wider, and includes also partially resonant solutions. The solutions described in this work represent the extreme case in which all of the interactions among the various solitons are resonant, whereas ordinary soliton solutions represent the opposite case where none of the interactions among the solitons are resonant. Inbetween these two situations, a number of intermediate cases exist in which only some of the interactions are resonant. As in the case of the KP equation, the study of these partially resonant solutions remains an open problem.

\section{Acknowledgements}

We thank M. J. Ablowitz, Y. Kodama, J. Matsukidaira and A. Nagai for many helpful discussions. K.M. acknowledges support from the Rotary foundation and the 21st Century COE program "Development of Dynamic Mathematics with High Functionality" at Faculty of Mathematics, Kyushu University. G.B. was partially supported by the National Science Foundation, under grant number DMS-0101476. 


\section{References}

[1] Biondini G and Kodama Y 2003 On a family of solutions of the Kadomtsev-Petviashvili equation which also satisfy the Toda lattice hierarchy, J. Phys. A 36, 10519-10536

[2] Hirota R, Ito M and Kako F 1988 Two-Dimensional Toda Lattice Equations, Prog. Theor. Phys. Suppl. 94, $42-58$

[3] Hirota R, Ohta Y and Satsuma J 1988 Wronskian structures of solitons for soliton equations, Prog. Theor. Phys. Suppl. 94, 59-72

[4] Isojima S, Willox R and Satsuma J 2002 On various solution of the coupled KP equation, J. Phys. A, Math. Gen. 35, 6893-6909

[5] Isojima S, Willox R and Satsuma J 2003 Spider-web solution of the coupled KP equation, J. Phys. A, Math. Gen. 36, 9533-9552

[6] Matsukidaira J, Satsuma J, Takahashi D, Tokihiro T and Torii M 1997 Toda-type cellular automaton and its $N$-soliton solution, Phys. Lett. A 225, 287-295

[7] Medina E 2002 An $N$ soliton resonance solution for the KP equation: interaction with change of form and velocity, Lett. Math. Phys. 62, 91-99

[8] Miles J W 1977 Diffraction of solitary waves, J. Fluid Mech. 79, 171-179

[9] Moriwaki S, Nagai A, Satsuma J, Tokihiro T, Torii M, Takahashi D and Matsukidaira J 1999 2+1 dimensional soliton cellular automaton London Math. Soc. Lecture Notes Series 255, Cambridge University Press, 334 342

[10] Nagai A, Tokihiro T, Satsuma J, Willox R and Kajiwara K 1997 Two-dimensional soliton cellular automaton of deautonomized Toda type, Phys. Lett. A 234, 301-309

[11] Newell A C and Redekopp L G 1977 Breakdown of Zakharov-Shabat theory and soliton creation, Phys. Rev. Lett. 38, 377-380

[12] Park J H H, Steiglitz K and Thurston W P 1986 Soliton-like behavior in automata, Physica D 19, 423-432

[13] Richter-Gebert J, Sturmfels B and Theobald T 2003 First steps in tropical geometry, arXiv:math.AG/ 0306366 ,

[14] Speyer D and Sturmfels B 2003 The tropical Grassmannian, arXiv:math. AG/0304218

[15] Speyer D and Williams L K 2003 The tropical totally positive Grassmannian, arXiv: math.CO/0312297

[16] Takahashi D and Matsukidaira J 1995 On discrete soliton equations related to cellular automata, Phys. Lett. A 209, 184-188

[17] Takahashi D and J. Satsuma J 1990 A soliton cellular automaton, J. Phys. Soc. Jpn. 59, 3514-3519

[18] Tokihiro T, Takahashi D, Matsukidaira J and Satsuma J 1996 From soliton equations to integrable cellular automata through a limiting procedure, Phys. Rev. Lett. 76, 3247-3250 


\section{List of MHF Preprint Series, Kyushu University 21st Century COE Program Development of Dynamic Mathematics with High Functionality}

\section{MHF2003-1 Mitsuhiro T. NAKAO, Kouji HASHIMOTO \& Yoshitaka WATANABE}

A numerical method to verify the invertibility of linear elliptic operators with applications to nonlinear problems

MHF2003-2 Masahisa TABATA \& Daisuke TAGAMI

Error estimates of finite element methods for nonstationary thermal convection problems with temperature-dependent coefficients

MHF2003-3 Tomohiro ANDO, Sadanori KONISHI \& Seiya IMOTO

Adaptive learning machines for nonlinear classification and Bayesian information criteria

MHF2003-4 Kazuhiro YOKOYAMA

On systems of algebraic equations with parametric exponents

MHF2003-5 Masao ISHIKAWA \& Masato WAKAYAMA

Applications of Minor Summation Formulas III, Plücker relations, Lattice paths and Pfaffian identities

MHF2003-6 Atsushi SUZUKI \& Masahisa TABATA

Finite element matrices in congruent subdomains and their effective use for large-scale computations

MHF2003-7 Setsuo TANIGUCHI

Stochastic oscillatory integrals - asymptotic and exact expressions for quadratic phase functions -

MHF2003-8 Shoki MIYAMOTO \& Atsushi YOSHIKAWA

Computable sequences in the Sobolev spaces

MHF2003-9 Toru FUJII \& Takashi YANAGAWA

Wavelet based estimate for non-linear and non-stationary auto-regressive model

MHF2003-10 Atsushi YOSHIKAWA

Maple and wave-front tracking - an experiment

MHF2003-11 Masanobu KANEKO

On the local factor of the zeta function of quadratic orders

MHF2003-12 Hidefumi KAWASAKI

Conjugate-set game for a nonlinear programming problem 
MHF2004-1 Koji YONEMOTO \& Takashi YANAGAWA

Estimating the Lyapunov exponent from chaotic time series with dynamic noise

MHF2004-2 Rui YAMAGUCHI, Eiko TSUCHIYA \& Tomoyuki HIGUCHI

State space modeling approach to decompose daily sales of a restaurant into time-dependent multi-factors

MHF2004-3 Kenji KAJIWARA, Tetsu MASUDA, Masatoshi NOUMI, Yasuhiro OHTA \& Yasuhiko YAMADA

Cubic pencils and Painlevé Hamiltonians

MHF2004-4 Atsushi KAWAGUCHI, Koji YONEMOTO \& Takashi YANAGAWA

Estimating the correlation dimension from a chaotic system with dynamic noise

MHF2004-5 Atsushi KAWAGUCHI, Kentarou KITAMURA, Koji YONEMOTO, Takashi YANAGAWA \& Kiyofumi YUMOTO

Detection of auroral breakups using the correlation dimension

MHF2004-6 Ryo IKOTA, Masayasu MIMURA \& Tatsuyuki NAKAKI

A methodology for numerical simulations to a singular limit

MHF2004-7 Ryo IKOTA \& Eiji YANAGIDA

Stability of stationary interfaces of binary-tree type

MHF2004-8 Yuko ARAKI, Sadanori KONISHI \& Seiya IMOTO

Functional discriminant analysis for gene expression data via radial basis expansion

MHF2004-9 Kenji KAJIWARA, Tetsu MASUDA, Masatoshi NOUMI, Yasuhiro OHTA \& Yasuhiko YAMADA

Hypergeometric solutions to the $q$ - Painlevé equations

MHF2004-10 Raimundas VIDŪNAS

Expressions for values of the gamma function

MHF2004-11 Raimundas VIDŪNAS

Transformations of Gauss hypergeometric functions

MHF2004-12 Koji NAKAGAWA \& Masakazu SUZUKI

Mathematical knowledge browser

MHF2004-13 Ken-ichi MARUNO, Wen-Xiu MA \& Masayuki OIKAWA

Generalized Casorati determinant and Positon-Negaton-Type solutions of the Toda lattice equation

MHF2004-14 Nalini JOSHI, Kenji KAJIWARA \& Marta MAZZOCCO

Generating function associated with the determinant formula for the solutions of the Painlevé II equation 
MHF2004-15 Kouji HASHIMOTO, Ryohei ABE, Mitsuhiro T. NAKAO \& Yoshitaka WATANABE

Numerical verification methods of solutions for nonlinear singularly perturbed problem

MHF2004-16 Ken-ichi MARUNO \& Gino BIONDINI

Resonance and web structure in discrete soliton systems: the two-dimensional Toda lattice and its fully discrete and ultra-discrete versions 